\title{
Risk of Acute Lymphoblastic Leukemia: Results of a Case-Control Study
}

\author{
Hassan Rafieemehr ${ }^{1 *}$, Fereshteh Calhor ${ }^{2}$, Hossein Esfahani ${ }^{3}$, Somayeh Ghorbani \\ Gholiabad $^{4}$
}

\begin{abstract}
Background: Acute lymphoblastic leukemia (ALL) is the most common malignancy in children. Different environmental factors might be effective in the occurrence of this malignancy during childhood. The aim of this study was to find environmental risk factors in childhood ALL in Hamadan, Iran. Methods: This case-control study was done in 2015-2018 on 125 children younger than 15 years of age suffering from ALL. Patients were matched with 130 controls with respect to age, gender, and residence location. The identification of risk factors for ALL was sought based on the comparison of studied variables between case and control individuals. Results: A statistically significant increased risk for ALL was found with regard to type of delivery (OR: 0.43, 95\% CI: $0.20-0.92, p<0.02$ ), childcare (OR: 4.58, 95\% CI: 0.95 - 22.20, p<0.04), birth weight (OR: 1.44, 95\% CI: $1.53-2.21, \mathrm{p}<0.006$ ), father's education level (OR: 2.67, 95\% CI: 1.10 - 6.45, p<0.02), and father's job (OR: 0.2 95\% CI: 0.08 - 0.51, p<0.001). Also observed were increased odds for ALL regarding male gender, mother's high education level, mother's freelance job, and medium or high family income. No association with ALL incidence was observed for age, gender, breastfeeding, mother's age at pregnancy, malignancy in first- or second-degree relatives, or mother's use of hair dye during pregnancy $(p>0.05)$. Conclusion: This study showed that father's education level, father's job, delivery type, birth weight, and childcare can play a role in the incidence of childhood ALL.
\end{abstract}

Keywords: Acute lymphoblastic leukemia- children- parents- risk factors

Asian Pac J Cancer Prev, 20 (8), 2477-2483

\section{Introduction}

Leukemias are malignant hematologic disorders characterized by the defective differentiation of immature white blood cells which leads to increased leukemic blasts cells in bone marrow (BM) and peripheral blood (PB) (Sant et al., 2010). Based on the cell type (lymphoid or myeloid) as well as the course of disease, leukemias are divided into acute forms, including acute lymphoblastic leukemia (ALL) and acute myeloid leukemia (AML), and chronic forms, including chronic myeloid leukemia (CML) and chronic lymphoblastic leukemia (CLL) (Sant et al., 2010; Vardiman et al., 2009). According to reports by the World Health Organization (WHO), ALL is the most common type of cancer in children younger than 15 years of age; it accounts for nearly one third of childhood cancers (Schrappe, 2018). Similar to other malignancies, ALL seems to be a multifactorial disease, and environmental and genetic factors interact with each other, playing a key role in its incidence (Eden, 2010). Several studies have demonstrated that environmental and genetic factors such as breastfeeding, keeping children in kindergarten, exposure to radiation, smoking, farm animals, and some inherited diseases (such as Fanconi anemia) can be associated with an increased risk for ALL in children (Gholami et al., 2011; Karimi et al., 2016; Moghaddasi et al., 2018; Mohammadi et al., 2018; Urayama et al., 2010). These findings suggest that knowing risk factors for childhood leukemia, especially ALL, is a crucial step in the prevention and reduction of ALL occurence in childhood. Despite the importance of environmental factors in ALL predispositions, few studies in Iran have examined the relationship between type of delivery, birth weight, breastfeeding experience, level of income, childcare history, parental education level, father's occupation, mother's age at pregnancy, and history of mother's use of hair dyes with the risk of this malignancy.

Considering the fact that several acquired and inherited factors play a role in the occurrence of leukemia, and given that there are various risk factors of ALL in different geographical regions (Hashemizadeh et al., 2013), this study investigated environmental characteristics as potential risk factors for ALL incidence among children less than 15 years old in Hamadan.

${ }^{1}$ Department of Medical Laboratory Sciences, School of Paramedicine, ${ }^{2}$ Students Research Center, ${ }^{3}$ Besat Hospital, ${ }^{4}$ Department of Biostatistics, School of Public Health, Hamadan University of Medical Sciences, Hamadan, Iran. *For Correspondence: rafee135298@gmail.com 


\section{Materials and Methods}

This matched case-control study was conducted on children with ALL who referred to Be'sat Hospital of Hamadan University of Medical Sciences during the years 2015-2018. Cases were children with a diagnosis of ALL confirmed through BM aspiration as well as flow cytometric analysis of the $\mathrm{PB}$ and $\mathrm{BM}$ cells. Controls were selected from children who referred to Be'sat Hospital for routine healthcare and had no previous history of invasive cancers or hematological malignancies. Controls and patients were frequency-matched by age, gender, and residence location at the time of the study. Informed consent was obtained from each individual participating in this study before data collection, in accordance with the institutional Ethics Committee of the Research Deputy of Hamadan University of Medical Sciences. Cases and controls were interviewed, and a questionnaire gathering detailed information on the variables under study was completed by the parents of each subject. The questionnaire included questions about age, gender, birth weight (high birth weight defined as $3,500 \mathrm{~g}$ or more), duration of breastfeeding, mother's age at pregnancy, educational level of parents, childcare, income level, father's occupation, smoking, type of delivery, history of mother's use of hair dyes, and percentage of malignancies in first- and second-degree relatives.

The inclusion criteria for cases were a diagnosis of ALL at the time of the study, the absence of a previous history of organ transplantation, age less than 15 years, and residence in Hamadan at the time of diagnosis.

Controls had no previous history of ALL, other invasive cancers, or hematological diseases and were frequency-matched to cases by age (less than 15 years), gender, race, and residence in Hamadan during data collection.

\section{Statistical analysis}

All statistical analyses were performed using SPSS version 22, and quantitative data was expressed as mean \pm SD. Logistic regression was used to estimate the odds ratios, and a $95 \%$ confidence interval was used to investigate the relationship between the studied variables and a predisposion to ALL. Chi-square and and t-tests for differences between groups were used to compare cases and controls to determine the association between the studied variables and the risk of ALL. A p-value of $<0.05$ was considered significant.

\section{Results}

One hundred twenty-five children with ALL and one hundred thirty healthy individual controls less than 15 years of age were enrolled in this study from 2015 to 2018. Out of the leukemic cases, 52 (41.6\%) were girls and $73(58.4 \%)$ were boys. Although the difference was not statistically significant, boys showed a 1.25 -time increase in odds for ALL (95\% CI: 0.61 - 2.57) than over

Table 1. ALL Incidence Risk based on Children Characteristics

\begin{tabular}{|c|c|c|c|c|}
\hline Characteristics & Cases $n=125$ & Controls $\mathrm{n}=130$ & Odds ratio $(95 \% \mathrm{CI})$ & $P$-value \\
\hline Sex; n (\%) & & & $1.25(0.61-2.57)$ & 0.54 \\
\hline Male & $73(58.4 \%)$ & $79(60.7)$ & & \\
\hline Female & $52(41.6 \%)$ & $51(39.3)$ & & \\
\hline Type of birth; n (\%) & & & $0.43(0.20-0.92)$ & 0.02 \\
\hline Caesarian & $89(71.2)$ & $68(52.4)$ & & \\
\hline Normal & $36(28.8)$ & $62(47.6)$ & & \\
\hline Birth weight (gr); n (\%) & & & $1.44(1.53-2.21)$ & 0.006 \\
\hline Mean \pm SD & $3130 \pm 2.1$ & $2510 \pm 1.4$ & & \\
\hline$\leq 2500$ & $16(12.8)$ & $20(15.3)$ & & \\
\hline $2,500-2,999$ & $21(16.8)$ & $36(27.3)$ & & \\
\hline $3,000-3,500$ & $31(24.8)$ & $61(46.9)$ & & \\
\hline$\leq 3,500$ & $57(45.6)$ & $14(10.5)$ & & \\
\hline Ever breast-fed; n (\%) & & & $0.02(0.54-1.93)$ & 0.57 \\
\hline No & $67(53.6)$ & $56(35.4)$ & & \\
\hline Yes & $58(46.4)$ & $74(64.6)$ & & \\
\hline \multicolumn{5}{|l|}{ Months breast-fed; n (\%) } \\
\hline $1-3$ & 20 & 23 & & \\
\hline $4-6$ & 13 & 17 & & \\
\hline $7-9$ & 11 & 13 & & \\
\hline $10-12$ & 8 & 10 & & \\
\hline$\geq 12$ & 6 & 11 & & \\
\hline Child care; n (\%) & & & $4.58(0.95-22.20)$ & 0.04 \\
\hline Home & $114(91.2)$ & $110(84.6)$ & & \\
\hline Kindergarten & $11(8.8)$ & $20(15.4)$ & & \\
\hline
\end{tabular}


girls. As shown in Table 1, the mean age of ALL patients was $6.4 \pm 5.23$ years (range, 1-10), and the mean age of control individuals was $7.43 \pm 8.11$ years (range, 1-12), but no significance difference was observed $(\mathrm{p}=0.74)$.

\section{Risk factors for ALL incidence}

To identify ALL risk factors, the association between different variables and ALL incidence was evaluated. Based on the children's characteristics, a significant association was found between both type of birth $(\mathrm{p}<0.02)$ and childcare $(\mathrm{p}<0.04)$ and increased risk of ALL (Table 1). High birth weight was also associated with an increased risk of ALL $(p<0.006)$ (Figure 1$)$. The risk of ALL was shown to be lower for children who were breastfed for more than 12 months, although no statistical significance was found $(\mathrm{p}<0.57)$. Univariable analysis showed that the odds of children delivered by caesarian for developing ALL was increased 0.43 times (95\% CI: 0.20 - 0.92) compared with children delivered vaginally. Moreover, an increased risk of ALL was observed among children whose fathers had a high level of education ( $\mathrm{p}$ $<0.02)$ or had a freelance job $(\mathrm{p}<0.001)$ (Table 2). The odds of developing ALL for children whose fathers had a higher level of education and those with a freelance job were 2.67 and 0.2 times higher than other children $(95 \%$ CI: 1.10 - 6.45), (95\% CI: 0.08 - 0.51), respectively.

Table 2. ALL Incidence Risk by Parental and Family Characteristics

\begin{tabular}{|c|c|c|c|c|}
\hline Characteristics & Cases $n=125$ & Controls $n=130$ & Odds ratio $(95 \% \mathrm{CI})$ & P-value \\
\hline Father's smoking; n (\%) & & & $0.50(0.22-1.13)$ & 0.09 \\
\hline Yes & $26(20.8)$ & $45(34.6)$ & & \\
\hline No & $99(79.2)$ & $85(65.4)$ & & \\
\hline Family income; $\mathrm{n}(\%)$ & & & $3.24(1.33-7.91)$ & 0.08 \\
\hline Low & $44(35.2)$ & $19(14.6)$ & & \\
\hline Medium or high & $81(64.8)$ & $111(85.4)$ & & \\
\hline Malignancy in first-degree relatives; $\mathrm{n}(\%)$ & & & $0.17(0.02-1.46)$ & 0.07 \\
\hline Yes & $9(7.2)$ & $12(9.3)$ & & \\
\hline No & $116(92.8)$ & $118(90.7)$ & & \\
\hline Malignancy in second-degree relatives; $\mathrm{n}(\%)$ & & & $0.94(0.44-1.98)$ & 0.87 \\
\hline Yes & $43(34.4)$ & $48(36.9)$ & & \\
\hline No & $82(65.6)$ & $82(63.1)$ & & \\
\hline Child care; $\mathrm{n}(\%)$ & & & $4.58(0.95-22.20)$ & 0.04 \\
\hline Home & $114(91.2)$ & $110(84.6)$ & & \\
\hline Kindergarten & $11(8.8)$ & $20(15.4)$ & & \\
\hline Father's education level; $\mathrm{n}(\%)$ & & & $2.67(1.10-6.45)$ & 0.02 \\
\hline$\leq$ High school & $84(67.2)$ & $86(66.1)$ & & \\
\hline$\geq$ High school & $41(32.8)$ & $44(33.9)$ & & \\
\hline Mother's education level; $\mathrm{n}(\%)$ & & & $1.55(0.65-3.66)$ & 0.32 \\
\hline$\leq$ High school & $101(80.8)$ & $95(73.1)$ & & \\
\hline$\geq$ High school & $24(19.2)$ & $35(26.9)$ & & \\
\hline Mother age at pregnancy, n (\%) & & & $1.48(0.31-0.6 .8)$ & 0.41 \\
\hline Mean of years \pm SD & $28.2 \pm 2.4$ & $25.6 \pm 3.7$ & & \\
\hline$\leq 19$ & $22(17.6)$ & $27(20.8)$ & & \\
\hline $20-25$ & $30(24.0)$ & $47(36.1)$ & & \\
\hline $26-30$ & $23(18.4)$ & $29(22.3)$ & & \\
\hline $31-35$ & $37(29.6)$ & $19(14.7)$ & & \\
\hline$\geq 35$ & $13(10.4)$ & $8(6.1)$ & & \\
\hline Father's job; n (\%) & & & $0.20(0.08-0.51)$ & 0.001 \\
\hline Employee & $16(12.8)$ & $54(41.5)$ & & \\
\hline Freelance & $109(87.2)$ & $76(58.5)$ & & \\
\hline Mother's job; n (\%) & & & $0.60(0.17-2.18)$ & 0.43 \\
\hline Employee & $9(7.2)$ & $15(11.5)$ & & \\
\hline Freelance & $116(92.8)$ & $115(88.5)$ & & \\
\hline \multicolumn{2}{|c|}{ Use of hair dye during pregnancy by mother; $\mathrm{n}(\%)$} & & $0.87(0.32-2.37)$ & 0.77 \\
\hline Yes & $18(14.4)$ & $21(16.1)$ & & \\
\hline No & $107(85.6)$ & $109(83.8)$ & & \\
\hline
\end{tabular}




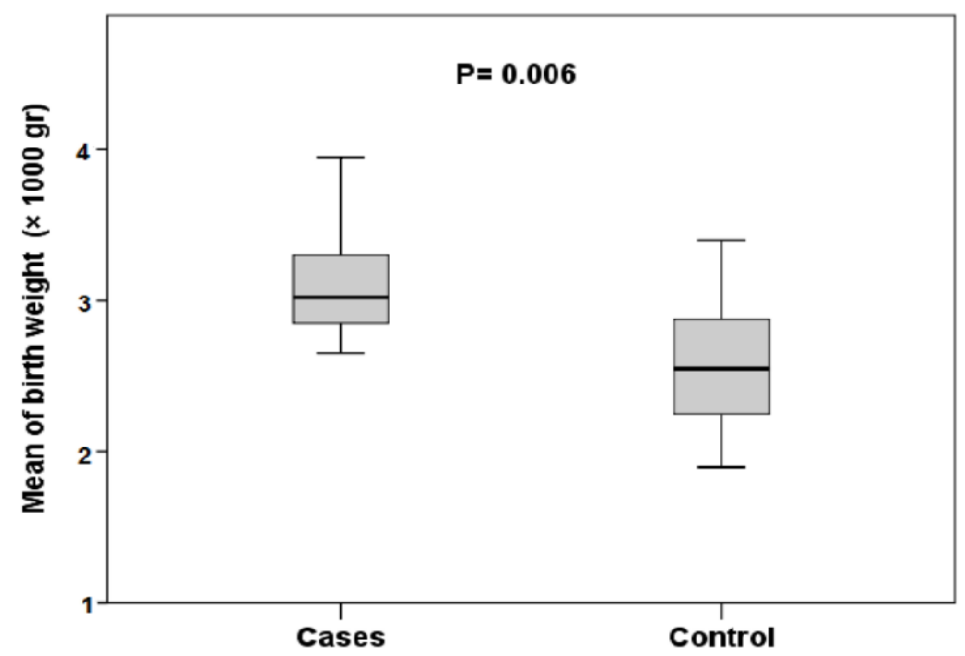

Figure 1. Comparison of Mean of Birth Weight between Cases and Control Individuals

Although no significant association was found between mother's education level or mother's job and ALL incidence, univariable analysis showed that the odds of children whose mothers had a high level of education or a freelance job were 1.55 and 0.6 times higher for developing ALL (95\% CI: 0.65 - 3.66), (95\% CI: 0.17 - 2.18), respectively. Similarly, a mother's high age at pregnancy was also associated with an increased risk for ALL, but the finding was not statistically significant (Figure 2). Children with homecare had 4.58 times higher odds for ALL occurrence (95\% CI: 0.95 - 22.20). No significant association was found between the risk for ALL and father's smoking, family income, malignancy in first- and second-degree relatives, or mother's use of hair dye during pregnancy (Table 2). However, the odds associated with family income showed that children with a medium- or high-level family income were 3.24 times higher for ALL incidence.

\section{Discussion}

In addition to cytogenetic abnormalities, the increased risk of ALL can be associated with different environmental factors. Several case-control studies have introduced heavy smoking, father's freelance job, exposure to low or moderate/high levels of pesticides, and obesity as the most common risk factors associated with the predisposition for ALL in children (Gholami et al., 2011; Moghaddasi et al., 2018; Mohammadi et al., 2018; Urayama et al., 2010). Among all enrolled cases in the current study, 52 (41.6\%) were girls and 73 (58.4\%) were boys. Although no significant difference between cases and control individuals was found to explain a predominant tendency to develop ALL, univariable analysis showed that boy's gender had higher odds for developing ALL. This finding is in agreement with previous studies (Gholami et al., 2011; Zakerinia et al., 2015). Since few studies have investigated the association between gender and development of ALL, whether this association may be an effect of sexual hormones is still unclear. The relationship between the development of ALL and lifestyle is another important issue. In the current study, a significant association was observed between both caesarian delivery and home childcare and the incidence of ALL in children. Similarly, Francis et al. and Marcotte et al. reported that because of the increased possibility of infection and the low level of immune responses with caesarian delivery, it is associated with the higher risk of ALL (Francis et al., 2014; Marcotte et al., 2016).

In the current study, high birth weight was associated

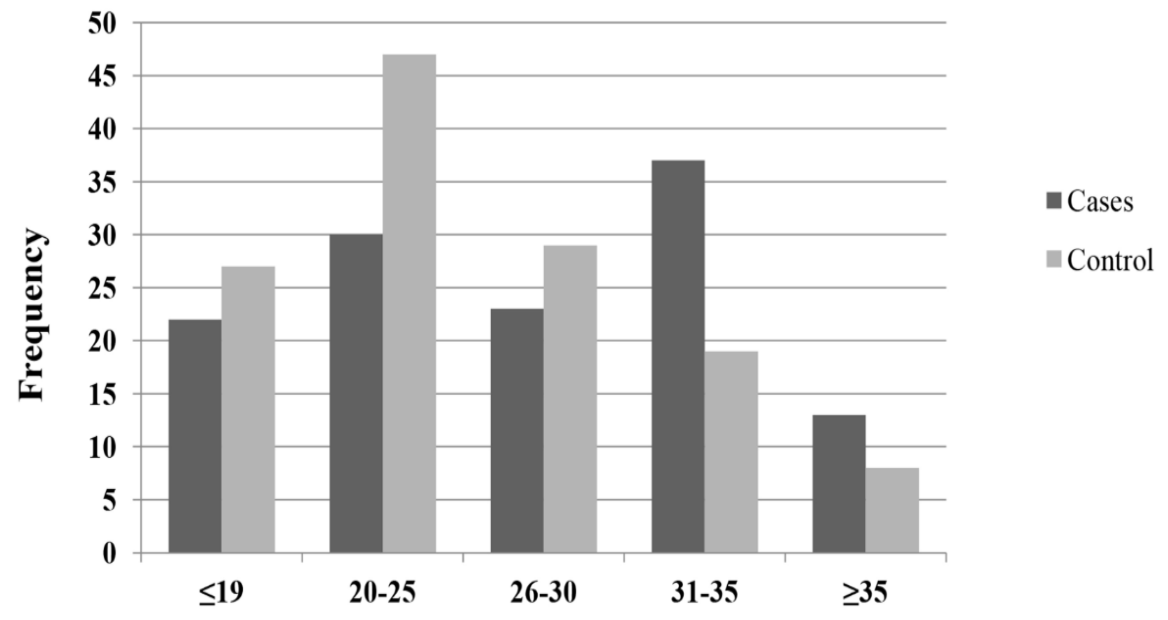

Figure 2. Frequency of Different Groups of Mothers Based on Age at Pregnancy 
with an increased risk of ALL. Similarly, this association has also been reported in recent large studies (Hjalgrim et al., 2003; McLaughlin et al., 2006; Oksuzyan et al., 2012; Von Behren et al., 2011); conversely, some studies have found a negative or weak increased risk of ALL among children who had higher birth weights (Ma et al., 2005; Shu et al., 2002). One possible mechanism suggested to explain this finding is insulin-like growth factor I (IGF-I), which is associated with the stimulation of myeloid and lymphoid cells proliferation (McLaughlin et al., 2006). Furthermore, maternal risk factors, including pregnancy weight gain, mother's own birth weight, diabetes, smoking, drug use, and duration of breastfeeding can also be related to higher birth weight as well as an increased risk for ALL (McLaughlin et al., 2006). Although this study was not able to account for either of these variables, an increased risk of ALL with a history of breastfeeding for less than 12 months was observed. Biologically possible mechanisms that may explain the relationship between breastfeeding and the increased risk of ALL relate to the fact that IGF1 level and its leukemogenesis effect have been isolated from human milk, while breast milk replacements do not have this capability (Martin et al., 2014).

Mother's high age at pregnancy is another risk factor for increased risk of ALL. However, no significant difference in risk for ALL between cases and control subjects was observed in this regard. There is some controversy in studies with regard to maternal age and the risk of ALL (Gholami et al., 2011; McLaughlin et al., 2006). While some studies have reported an increased risk of ALL in children of mothers with a high age at pregnancy, numerous studies have shown a negative or weak risk of ALL with older maternal age (Jourdan-Da Silva et al., 2004; Murray et al., 2002; Okcu et al., 2002). As leukemia is a disease related to chromosomal breakage and translocation, older maternal age, which is associated with an increased risk of chromosomal malformations, is suggested as a potential risk factor for ALL incidence (McLaughlin et al., 2006). In the censored analysis in which childcare was assessed, it was found that a large number of participants were under home care. Published reports concerning the role of childcare practices in childhood ALL are few; however, Ma et al., (2002) suggested that exposure to more children and common infections at daycare play a vital role in the increased risk of ALL. Although this finding was quite unexpected for authors, the hypothesis herein is that parents of the cases in this study possessed limited information and experiences about home childcare. In contrast to the results of Shu and Wen who reported a negative correlation between parents' education level and ALL incidence (Shu et al., 1999; Wen et al., 2000), in the current study, it was observed that children who had parents with high education levels had increased odds for ALL incidence. However, some studies have not reported a significant correlation between parents' education level and childhood leukemia (Zolala et al., 2004). In the current study, it was observed that children with a medium or high family income had increased odds for ALL. It is inferred that because parents are working, they have less time to spend caring for their children. In addition, the increased odds for ALL incidence in children whose mothers had high education levels or freelance jobs in the current study may be related to the low duration of breastfeeding that was introduced as a risk factor for childhood leukemia ( Martin et al., 2005; Shu et al., 1999). Similar to mother's job, more than two thirds of the participants in the current study had fathers who had a freelance job, and it was found that these children had higher odds for developing ALL than children who had employed fathers. This finding confirmed those of previous studies that have identified father's freelance job (such as animal husbandry, agriculture, work in the rubber industry, and work in an oil refinery) as a common risk factor for childhood ALL (Chen et al., 2006; Hassanzade et al., 2012; Mehrabani et al., 2008). This higher incidence of ALL in children whose fathers had a freelance job may be attributed to the higher exposure to chemical materials, such as pesticides or insecticides, and contact with animals that are a threat to human health (Infante-Rivard et al., 1999; Zakerinia et al., 2015). In the present study, fathers of cases were more occupationally exposed to pesticides containing benzene. Although occupational exposure to benzene is one of the most consistently reported risk factors for hematologic malignancies, solid tumors, and lung dysfunction (Hesam et al., 2019; Panahi et al., 2018; Samadi et al., 2019; Schnatter et al., 2012), no significant differences were observed between exposure to pesticides and other chemical materials, including dyes, mineral oils, motor vehicle oils, and gasoline. It is assumed that exposure to pesticides was more likely to be related with ALL incidences in cases of the current study. In our assessment of relationship between father's smoking and risk of ALL, No significant relation between father's smoking and risk of ALL was found in the current study, which is the most considerable difference between this study and previous literature findings that have reported a positive association (Damirchi et al., 2013; Kasim et al., 2005). However, in agreement with the current findings, Bjork et al. reported that there was no significant association between smoking and AML risk (Björk et al., 2009). Since numerous epidemiologic studies have demonstrated the association between smoking and both childhood and adult leukemias, it may be that the small sample size in the current study reduced the statistical significance of the relationship between this important risk factor and ALL incidence. In contrast to the meta-analysis by Veisani et al. that showed that the first relative history of leukemia was associated with an increase of four times the risk of leukemia in children aged less than 15 years old (Veisani et al., 2017), no significant association between it and a history of a malignancy in first- and second-degree relatives was not found in the current study. Because the major percentage of cases and controls in the present study had no family history of malignancies, it seems that environmental factors have potential influences for incidence of ALL in Hamadan. The use of hair dye which contains numerous carcinogenic and mutagenic chemicals is another factor that might increase the risk of developing leukemia. However, in disagreement with other case-control studies that have shown that hair dyes may have an impact on the risk of adult acute leukemia (Rauscher et al., 2004; Towle et al., 2017), in this study, 
no significant association was found between mother's use of hair dye during pregnancy and the risk of ALL. It may be that the impact of dyes on ALL incidence is related to the duration and frequency of use or to the amounts of carcinogenic and mutagenic chemical components in the dyes.

In conclusion, in this case-control study, multiple risk factors for ALL incidence in children were identified including differences in types of birth, higher weight at birth, childcare, father's education level, and father's freelance job. The current study also suggests the increased odds for ALL can be associated with mother's higher education level, mother's freelance job, mother's high age at pregnancy, low duration of breastfeeding, boys gender, and a meduim or high family income. These findings highlight the importance of identifying epidemiological risk factors in addition to the genetic background to better understand the underlying heterogeneity of ALL. Because of the limited number of individuals in this study, identifying other childhood ALL risk factors was difficult. Therefore, more research with a higher number of participants is necessary to accurately detect ALL risk factors.

\section{Acknowledgments}

We wish to thank all our colleagues in Hamadan University of Medical Sciences.

\section{Authors' contributions}

H.R. has conceived the manuscript and revised it. H.E provided clinical data and information. H.R., F.C. and S.G.G performed the statistical analysis, wrote the manuscript, and prepared tables and figures.

\section{Funding/Support}

This study was funded by Vice-chancellor for Research and Technology, Hamadan University of Medical Sciences (No: 9507134142).

Research involving human participants and/or animals

All procedures performed in studies involving human participants were approved by the institutional ethics committee of Research Deputy of Hamadan University (IR.UMSHA.REC.1395.311).

\section{Informed consent}

Informed consent was obtained from all individual participants included in the study.

\section{Conflict of interest}

All authors approved the manuscript and have no declaration regarding commercial or non-commercial intentions.

\section{References}

Björk J, Johansson B, Broberg K, Albin M (2009). Smoking as a risk factor for myelodysplastic syndromes and acute myeloid leukemia and its relation to cytogenetic findings: A case-control study. Leuk Res, 33, 788-91.

Chen Z, Robison L, Giller R, et al (2006). Environmental exposure to residential pesticides, chemicals, dusts, fumes, and metals, and risk of childhood germ cell tumors. Int $J$ Hyg Environ Health, 209, 31-40.

Damirchi P, Soltani M, Bahrami Z, et al (2013). Assessment of the relation between pediatrics lymphohematopoietic cancers and Cigarette smoking parents. Bull Env Pharmacol Life Sci, 2, 10-3.

Eden T (2010). Aetiology of childhood leukaemia. Cancer Treat Rev, 36, 286-97.

Francis SS, Selvin S, Metayer C, et al (2014). Mode of delivery and risk of childhood leukemia. Cancer Epidemiol Biomarkers Prev, 23, 876-81.

Gholami A, Salarilak S, Hejazi S, Khalkhali HR (2011). Parental risk factors of childhood acute leukemia: a case-control study. J Res Health Sci, 11, 69-76.

Hashemizadeh H, Boroumand H, Noori R, Darabian M (2013). Socioeconomic status and other characteristics in childhood leukemia. Iran J Ped Hematol Oncol, 3, 182-86.

Hassanzade J, Mohammadi R, Rajaeefard A (2012). Risk factors in childhood lymphoblastic leukemia in Shiraz-Iran (2009): an epidemilogical study. J Gorgan Uni Med Sci, 14, 119-24.

Hesam M, Shakerkhatibi M, Samadi MT, et al (2019). Long-term exposure to outdoor VOCs and lung function in urban adults: a cross-sectional study in Tabriz an industrialized city in the northwest of Iran. Human and Ecolog Risk Assess Intl J, 25, 1-17.

Hjalgrim LL, Westergaard T, Rostgaard K, et al (2003). Birth weight as a risk factor for childhood leukemia: a meta-analysis of 18 epidemiologic studies. Am J Epidemiol, 158, 724-35.

Infante-Rivard C, Labuda D, Krajinovic M, Sinnett D (1999). Risk of childhood leukemia associated with exposure to pesticides and with gene polymorphisms. $J$ Epidemiol, 10, 481-7.

Jourdan-Da Silva N, Perel Y, Méchinaud F, et al (2004). Infectious diseases in the first year of life, perinatal characteristics and childhood acute leukaemia. Br J Cancer, 90, 139.

Karimi M, Haghighat M, Dialameh Z, et al (2016). Breastfeeding as a protective effect against childhood leukemia and lymphoma. Iran Red Crescent Med J, 18, e29771.

Kasim K, Levallois P, Abdous B, Auger P, Johnson KC (2005). Lifestyle factors and the risk of adult leukemia in Canada. Cancer Causes Control, 16, 489-500.

Ma X, Buffler P, Selvin S, et al (2002). Daycare attendance and risk of childhood acute lymphoblastic leukaemia. $\mathrm{Br} J$ Cancer, 86, 1419.

Ma X, Metayer C, Does MB, Buffler PA (2005). Maternal pregnancy loss, birth characteristics, and childhood leukemia (United States). Cancer Causes Control, 16, 1075-83.

Marcotte EL, Thomopoulos TP, Infante-Rivard C, et al (2016). Caesarean delivery and risk of childhood leukaemia: a pooled analysis from the Childhood Leukemia International Consortium (CLIC). Lancet Haematol, 3, 176-85.

Martin F-PJ, Moco S, Montoliu I, et al (2014). Impact of breast-feeding and high-and low-protein formula on the metabolism and growth of infants from overweight and obese mothers. Ped Res, 75, 535.

Martin RM, Gunnell D, Owen CG, Smith GD (2005). Breastfeeding and childhood cancer: a systematic review with metaanalysis. Int J Cancer, 117, 1020-31.

McLaughlin C, Baptiste M, Schymura M, Nasca P, Zdeb M (2006). Birth weight, maternal weight and childhood leukaemia. Br J Cancer, 94, 1738.

Mehrabani D, Tabei S, Heydari S, et al (2008). Cancer occurrence in Fars province, southern Iran. Iran Red Crescent Med J, 10, 314-22.

Moghaddasi J, Taleghani F, Moafi A, et al (2018). Family interactions in childhood leukemia: an exploratory 
descriptive study. Support Care Cancer, 26, 4161-68.

Mohammadi M, Naderi M, Ansari Moghaddam A, Mahdavifar N, Mohammadian M (2018). Investigation of the relationship between breastfeeding and leukemia in children. Iranian $J$ Ped Hematol Oncol, 8, 97-104.

Murray L, McCarron P, Bailie K, et al (2002). Association of early life factors and acute lymphoblastic leukaemia in childhood: historical cohort study. Br J Cancer, 86, 356.

Okcu MF, Goodman KJ, Carozza SE, et al (2002). Birth weight, ethnicity, and occurrence of cancer in children: a population-based, incident case-control study in the State of Texas. Cancer Causes Control, 13, 595-602.

Oksuzyan S, Crespi C, Cockburn M, Mezei G, Kheifets L (2012). Birth weight and other perinatal characteristics and childhood leukemia in California. Cancer Epidemiol, 36, 359-65.

Panahi Y, Alikhani MY, Rafiee M, et al (2018). Prostate specific antigen level in exposure to sulfur mustard. Iran J Blood Cancer, 9, 121-4.

Rauscher GH, Shore D, Sandler DP (2004). Hair dye use and risk of adult acute leukemia. Am J Epidemiol, 160, 19-25.

Samadi MT, Shakerkhatibi M, Poorolajal J, et al (2019). Association of long term exposure to outdoor volatile organic compounds (BTXS) with pro-inflammatory biomarkers and hematologic parameters in urban adults: A cross-sectional study in Tabriz, Iran. Ecotoxicol Environ Saf, 180, 152-9.

Sant M, Allemani C, Tereanu C, et al (2010). Incidence of hematologic malignancies in Europe by morphologic subtype: results of the haemacare project. Blood, 116, 3724-34.

Schnatter AR, Glass DC, Tang G, Irons RD, Rushton L (2012). Myelodysplastic syndrome and benzene exposure among petroleum workers: an international pooled analysis. J Natl Cancer Inst, 104, 1724-37.

Schrappe M (2018). Acute lymphoblastic leukemia. HemaSphere, 2,1 .

Shu XO, Linet MS, Steinbuch M, et al (1999). Breast-feeding and risk of childhood acute leukemia. J Natl Cancer Inst, 91, 1765-72.

Shu XO, Han D, Severson RK, et al (2002). Birth characteristics, maternal reproductive history, hormone use during pregnancy, and risk of childhood acute lymphocytic leukemia by immunophenotype. Cancer Causes Control, 13, 15-25.

Towle KM, Grespin ME, Monnot AD (2017). Personal use of hair dyes and risk of leukemia: a systematic literature review and meta-analysis. Cancer Med, 6, 2471-86.

Urayama KY, Buffler PA, Gallagher ER, Ayoob JM, Ma X (2010). A meta-analysis of the association between day-care attendance and childhood acute lymphoblastic leukaemia. Int $J$ Epidemiol, 39, 718-32.

Vardiman JW, Thiele J, Arber DA, et al (2009). The 2008 revision of the World Health Organization (WHO) classification of myeloid neoplasms and acute leukemia: rationale and important changes. Blood, 114, 937-51.

Veisani Y, Delpisheh A (2017). A meta-analysis of prenatal and maternal risk factors for childhood leukemia in iran, casecontrol studies approach. J Pediatr Rev, 10, e14674.

Von Behren J, Spector LG, Mueller BA, et al (2011). Birth order and risk of childhood cancer: a pooled analysis from five US States. Int J Cancer, 128, 2709-16.

Wen W-Q, Shu X-O, Steinbuch M, et al (2000). Paternal military service and risk for childhood leukemia in offspring. $\mathrm{Am} \mathrm{J}$ Epidemiol, 151, 231-40.

Zakerinia M, Amirghofran S, Namdari, et al (2015). Relationship between exposure to pesticides and occurrence of acute leukemia in Iran. Asian Pac J Cancer Prev, 16, 239-44.
Zolala F, Ayatollahi S, Ayatollahi S, Shahriary M (2004). Determination the inducing factors of acute lymphoblastic leukemia in children under 15 years old in Fars province in the year 2001. J Rafsanjan Uni Med Sci, 3, 267-75.

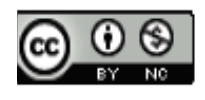

This work is licensed under a Creative Commons AttributionNon Commercial 4.0 International License. 